\title{
A Branch and Bound Methodology for Matrix Polytope Stability Problems Arising in Power Systems
}

\author{
C. L. DeMarco \\ Department of Electrical and Computer Engineering \\ University of Wisconsin-Madison \\ Madison, WI 53706
}

\author{
V. Balakrishnan and S. Boyd \\ Information Systems Laboratory \\ Department of Electrical Engineering \\ Stanford University \\ Stanford, CA 94305
}

\begin{abstract}
This paper proposes a formulation to provide necessary and sufficient conditions for robust stability of a family of matrices modeling linearized power system dynamics. A construction for transforming variations in operating point into a polytope of matrices for the linearized system models is derived. Sufficient conditions to establish robust stability or instability of a polytope are developed. Finally, an iterative "branch and bound" technique is described that combines these tests to provide a necessary and sufficient test for robust stability of the matrix polytope.
\end{abstract}

\section{Introduction and Background}

Power system planners face the problem of designing control and operation schemes to ensure that the dynamics of the network remain well behaved over a wide range of operating points. As customer load levels vary, the system equilibrium moves quasistatically to track these changes; as a minimal requirement for acceptable operation, the eigenvalues associated with the family of linearizations about these equilibria should remain strictly in the open left half of the complex plane. For example, bifurcation phenomena associated with recent voltage collapse failures in power networks have raised concerns that operating conditions may evolve to a stage where the equilibrium disappears, indicating that an eigenvalue of the linearized system has gone to the origin. Computationally efficient tests to identify such undesirable operating regimes, and to guarantee acceptable linearized dynamics over a family of operating points, have become increasing important in power system planning.

For the purposes of this paper, a set $S$ of nxn real matrices representing linearized power system dynamics will be classified as "robustly stable" if for every $\mathbf{A} \in \mathcal{S}$, the spectrum of $\mathbf{A}$ is contained in the open left half plane. If there exists an $A \in S$ with an eigenvalue having non-negative real part, the set will be classified as unstable. This paper will first develop a dynamic model and a method of parameterizing uncertainty in the operating points for power systems such that the resulting set of linearized dynamic matrices forms a polytope. An algorithm to classify the resulting polytope as either robustly stable or unstable is then developed. The basic building blocks of the algorithm will be a sufficiency test for establishing robust stability, and another for establishing instability. Roughly speaking, the algorithm attempts to "bound" a given polytope to classify it as robustly stable or unstable. Should both tests fail, the polytope is halved, and the algorithm "branches" to apply the sufficiency tests to the two subpolytopes. The nature of the tests is such that ultimately one or the other must succeed when the sub-polytopes are sufficiently small. The branching to create successively smaller sub-polytopes continues until one proves to be unstable, or all can be characterized as robustly stable.

\section{Polytope Parameterization for Linearized Power System Models}

A description of a set of linearized system matrices for a robust stability test involves a trade-off between computational tractability of the problem versus accurate representation of physical phenomena that introduce uncertainty. The most significant parameters influencing a power system operating point are changes in aggregate load demand at distribution substations. A straightforward approach might specify a set of possible system loads as given data, and a hypothetical robust stability algorithm could then compute the resulting family of operating points, the linearized system dynamics for each of those operating points, and test for acceptable eigenvalue locations in these linearizations. Current utility practice approximates this approach, but with the significant drawback that Monte-Carlo sampling among the possible load vectors is used, often with some attempt at reducing the cost of repeated eigenvalue computations for each of the matrices for the resulting linearized dynamics [1]. The drawbacks of such an approach include the obvious computational cost in system models that can contain thousands of states, and the high sensitivity of eigenvalue location to the system matrix elements. Unless such tests are coupled with a measure of eigenvalue conditioning to determine how "fine" a sample must be examined, there is the risk that all sampled points may be prove acceptable, while other operating points in the set yield linearizations with unstable eigenvalues.

To ease the computational burden in the robust stability test, the approach proposed here parameterizes uncertainty in a different but physically meaningful way. Rather than taking bus power 
injections as the uncertain parameters, this formulation will use the voltage phase angle differences across each transmission line, denoted as $\sigma$, as the underlying uncertain parameters. We assume that the given data will consist of upper and lower bounds on these "line angles" over the range of operating points of interest. The observant reader will note that by treating line angles as independent uncertain parameters, a degree of conservatism will be introduced in the analysis. For a network of $\ell$ lines, basic network theory implies that line angles cannot vary arbitrarily over a hyper-rectangle in $\mathbf{R}^{\ell}$; indeed, $\mathrm{KVL}$ implies that the set of possible line flows must lie in a lower dimensional subspace. However, for computational convenience, we will accept this over-bounding. Hence, the given data parameterizing uncertainty in the operating point will be assumed to have the form:

$$
\sigma_{k} \in\left[\sigma_{k}^{\min }, \sigma_{k}^{\max }\right], k=1,2, \ldots, l .
$$

The simple "classical" model for the power system dynamics will be considered here. While practical application of robust stability tests will typically focus on much more detailed representations, this model is adequate to illustrate the components of the system linearization affected by changes in operating point. The classical model reflects the second order rotational dynamics of each generator, as coupled through the transmission network. The underlying dynamics are of the form inertia \{rotational acceleration $\}=$ net torque. For readers not familiar with power systems modeling, the key assumptions to recognize are that voltage frequency of a generator is proportional to rotational speed, and electrical power delivered is linearly related to torque. Hence the dynamics can be re-written as \{derivative of frequency\} $=\{\text { inertia }\}^{-1} \cdot$ (net power $)$ and (derivative of voltage phase angle $]$ $=\{$ frequency $\}$. More precisely::

$$
\begin{aligned}
& \dot{\omega}=M^{-1}\{D \omega+T(P-f(\alpha))\} \\
& \dot{\alpha}=T^{T} \omega
\end{aligned}
$$

where

$\omega=$ bus frequencies,

$\alpha=$ bus voltage phase angles,

$\mathbf{M}=$ diagonal matrix of positive generator inertia constants,

D = diagonal matrix of positive generator damping constants,

$\mathrm{f}_{\mathrm{i}}(\alpha)=$ power absorbed by network at bus $\mathrm{i}=$

$$
\sum_{k=1}^{n} b_{i k} \sin \left(\alpha_{i}-\alpha_{k}\right)+g_{i k} \cos \left(\alpha_{i}-\alpha_{k}\right)
$$

$b_{i k}=$ imaginary part of admittance linking buses $i-k$ $\mathrm{g}_{\mathrm{ik}}=$ real part of admittance linking buses $\mathrm{i}-\mathrm{k}$

$P_{i}=$ mechanical power delivered to shaft of generator $i$

$$
\begin{aligned}
\mathbf{T} & =\left[\begin{array}{l}
\mathbf{I}_{\mathrm{n}-1 \times \mathrm{n}-1} \\
-\mathbf{e}^{\mathrm{T}}
\end{array}\right], \\
\mathbf{e} & \in \mathbf{R}^{\mathrm{n}-1} ; \mathbf{e}=[-1,-1, \ldots,-1]^{\mathrm{T}} .
\end{aligned}
$$

Note that the dependence of the linearization of (1) on changes in operating point will be entirely determined by the structure of the Jacobian of $\mathbf{f}$, denoted here as $\mathbf{J}(\alpha)$. In particular, the linearization of these dynamics will take the form:

$$
\left[\begin{array}{cc}
\mathbf{M}^{-1} \mathbf{D} & -\mathbf{M}^{-1} \mathbf{T J}(\alpha) \\
\mathbf{T}^{T} & 0
\end{array}\right] .
$$

Note that $\mathbf{M}, \mathbf{D}$, and $\mathbf{T}$ are constant matrices that do not change with operating point. While more detailed models will introduce additional states with corresponding state equations, the variation in the linearization with respect to changes in operating point will almost always involve a block of the form (constant matrix) $\cdot J(\alpha)$.

Recall that the goal in this formulation is to relate the structure of $\mathbf{J}$ to the assumed uncertainty in line angles. The approach exploits some standard concepts of basic circuit theory. We associate with the power network a directed graph; each transmission line is associated with a branch and each bus with a node of the di-graph. The orientation of each branch can be assigned arbitrarily. Let

$$
\text { A := incidence matrix for the reduced network [2]. }
$$

The vector of phase angle differences across each line is then given by:

$$
\sigma=\mathbf{A}^{\mathrm{T}} \alpha .
$$

This confirms the earlier observation that a hyper-rectangle over bounds the physically achievable line angle variations, because these must lie in the range of $\mathbf{A}^{\mathrm{T}}$.

With this notation, $f(\alpha)$ from (1) may be re-written as a function of the line angles, i.e.

$$
\left.\left.f(\sigma)=\mathbf{A} \mid \begin{array}{c}
\mathrm{b}_{1} \sin \left(\sigma_{1}\right) \\
\mathrm{b}_{2} \sin \left(\sigma_{2}\right) \\
\vdots \\
\vdots \\
\mathrm{b}_{\ell} \sin \left(\sigma_{\ell}\right)
\end{array}\right]+|\mathbf{A}| \begin{array}{c}
\mathrm{g}_{1} \cos \left(\sigma_{1}\right) \\
\mathrm{g}_{2} \cos \left(\sigma_{2}\right) \\
\vdots \\
\vdots \\
\mathrm{g}_{\ell} \cos \left(\sigma_{\ell}\right)
\end{array}\right]
$$

$b_{k}=b_{i j}=b_{j i}$ if branch $k$ links bus $i$ to $j$, zero otherwise; $\mathrm{g}_{\mathrm{k}}=\mathrm{g}_{\mathrm{ij}}=\mathrm{g}_{\mathrm{ji}}$ if branch $\mathrm{k}$ links bus $\mathrm{i}$ to $\mathrm{j}$, zero otherwise; IAl denotes the matrix formed by taking absolute value of each element of $\mathbf{A}$.

The desired Jacobian expressed as a function of $\sigma$ becomes:

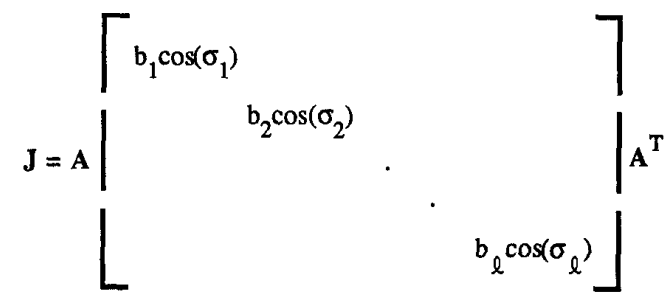


$\left.+|A| \mid \begin{array}{llll}-g_{1} \sin \left(\sigma_{1}\right) & & \\ & -g_{2} \sin \left(\sigma_{2}\right) & & \\ & & \cdot & \\ & & & -g_{\ell} \sin \left(\sigma_{\ell}\right)\end{array}\right]$

(2)

The diagonal sine and cosine dependence in (2) indicate that a hyper-rectangle in the space of line angles will not directly yield a polytope set of Jacobian matrices. However, the simple form of the dependence on the $\left(b_{\mathfrak{i}} \cos \left(\sigma_{\mathfrak{i}}\right),-\mathrm{g}_{\mathbf{i}} \sin \left(\sigma_{\mathfrak{i}}\right)\right)$ pairs suggests an approximation that will over-bound the true set. The resulting degree of conservatism will be small in cases where the transmission line losses are small relative to the line susceptance (i.e., $g_{\mathfrak{i}} \ll b_{i}$ ). Let

$$
z_{i}=b_{i} \cos \left(\sigma_{i}\right), z_{i+l}=-g_{i} \sin \left(\sigma_{i}\right), \text { for } i=1,2, \ldots, l,
$$

and

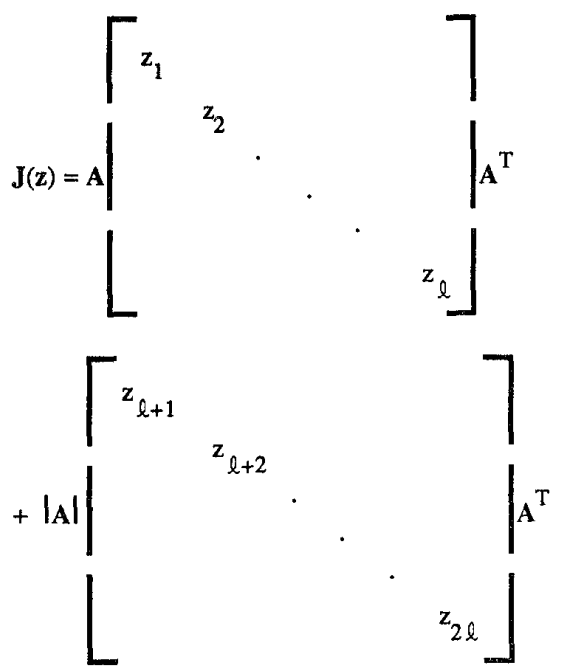

As each $\sigma_{i}$ varies over an interval, the $\left(b_{i} \cos \left(\sigma_{i}\right),-g_{i} \sin \left(\sigma_{i}\right)\right)$ pair maps to a curve in $\mathbf{R}^{2}$. Our approach will simply indentify the rectangle for $\left(z_{i}, z_{\ell+i}\right)$ in $\mathbf{R}^{2}$ that contains this curve. The polytope of feasible $\mathbf{z}$ in $\mathbf{R}^{2 \ell}$ will then form a hyper-rectangle, denoted as $\tilde{Z}$. Since $J(z)$ is linear with respect to $z$, the image $\mathbf{J}(\widetilde{Z})$ will form a polytope. For the analysis to follow, it will prove convenient to exploit this linearity to express $J(\mathbf{z})$ as a weighted sum of constant matrices $\mathbf{J}^{\mathrm{i}}$ :

$$
\mathbf{J}(\mathbf{z})=\sum_{\mathrm{i}=1}^{2 \ell} z_{\mathrm{i}} \mathbf{J}^{\mathrm{i}}
$$

To express the family of system linearizations as a weighted sum, it will prove convenient to expand the dimension of the hyper-rectangle by one. This additional axis is contracted to a degenerate interval of a single point, $z_{2 \ell+1} \equiv 1$ The resulting
$2 \ell+1$ dimensional hyper-rectangle will be denoted as $\tilde{Z}$ (single tilde). The family of linearizations becomes:

$$
L(z)=\sum_{i=1}^{2 \ell+1} z_{i} L^{i}
$$

where

$$
\mathbf{L}^{\mathrm{i}}=\left[\begin{array}{cc}
\mathbf{0} & -\mathbf{M}^{-1} \mathbf{T} \mathbf{J}^{\mathrm{i}} \\
\mathbf{0} & \mathbf{0}
\end{array}\right] \text { for } \mathrm{i}=1,2, \ldots 2 \ell ; \mathbf{L}^{2 \ell+1}=\left[\begin{array}{cc}
\mathbf{M}^{-1} \mathbf{D} & \mathbf{0} \\
\mathbf{T}^{\mathrm{T}} & 0
\end{array}\right]
$$

\section{Sufficient Condition for Robust Stability of a Matrix Polytope}

Given a polytope of matrices, the numerical analysis and control systems literature documents a wide variety of sufficient conditions for guaranteeing that each matrix in the polytope has all eigenvalues in the open left half plane; see, for example, [3] and the references therein. The test employed here is chosen to be easily adapted to the model structure described above with low computational cost. The price is a high degree of conservatism; a polytope can easily fail the sufficient condition below, and yet all the matrices in that polytope may be stable. For overall stability algorithm to be proposed in Section V, the consequence will be that a polytope may need to be divided into a larger number of smaller sub-polytopes than would be necessary with a less conservative test. However, the low computational cost for each application of the test was judged to outweigh this potential drawback.

The approach for establishing stability employes a simple matrix norm bound on eigenvalue perturbation. Let $z_{i}^{c} \triangleq$ $\left(z_{i}^{\max }+z_{i}^{\min }\right) / 2$ represent the center point of each of the given intervals in $\mathbf{z}$, and $\mathbf{L}\left(\mathbf{z}^{\mathrm{c}}\right)$ the corresponding linearized system matrix. $\mathbf{L}\left(\mathbf{z}^{\mathrm{c}}\right)$ is assumed diagonalizable, so there exists a $\mathbf{H} \in \mathbf{C}^{\text {nxn }}$ such that $\mathbf{H}^{-1} \mathbf{L}\left(\mathbf{z}^{\mathfrak{c}}\right) \mathbf{H}=\operatorname{diag}\left(\lambda_{\mathrm{i}}\right)$. Moreover, $\mathbf{L}\left(\mathbf{z}^{\mathcal{c}}\right)$ must be stable (if not, the polytope is obviously classified as unstable), with "minimum stability degree" defined as

$$
\delta_{0}^{\mathfrak{c}}=-\max \operatorname{Re}\left\{\lambda_{\mathrm{i}}\left(\mathbf{L}\left(\mathbf{z}^{\mathfrak{c}}\right)\right)\right\} .
$$

Exploiting the linearity of $L(z)$ in (4), one has

$$
\begin{aligned}
\mathbf{L}(\mathbf{z}) & =\mathbf{L}\left(\mathbf{z}^{\mathfrak{c}}\right)+\mathbf{L}\left(\mathbf{z}-\mathbf{z}^{\mathfrak{c}}\right) \\
& =\mathbf{L}\left(\mathbf{z}^{\mathfrak{c}}\right)+\left[\begin{array}{cc}
\mathbf{0} & \mathbf{M}^{-1} \mathbf{T} \mathbf{J}\left(\mathbf{z}-\mathbf{z}^{\mathfrak{c}}\right) \\
\mathbf{0} & 0
\end{array}\right] .
\end{aligned}
$$

The following Lemma bounds the norm variation in $\mathbf{L}$ as $\mathbf{z}$ varies from the "center" point.

$$
\begin{aligned}
& \text { Lemma } 1 \\
& \quad\left\|\mathbf{L}\left(\mathbf{z}-\mathbf{z}^{\mathfrak{c}}\right)\right\|_{1} \\
& \quad \leq 4 \cdot\left\|\mathbf{A}^{\mathrm{T}}\right\|_{1} \cdot \max _{1 \leq i \leq \mathbf{n}} M_{i}^{-1} \cdot\left\{\max _{1 \leq j \leq \ell}\left(z_{j}-z_{j}^{c}\right)+\max _{1 \leq k \leq 2 \ell}\left(z_{k}-z_{k}^{c}\right)\right\}
\end{aligned}
$$


Proof

From the definition of $\mathbf{J}(\mathbf{z})$ in (3) it follows that

$$
\|\mathbf{J}(\mathbf{z})\|_{1} \leq\|\mathbf{A}\|_{1} \cdot\left\{\max _{1 \leq i \leq \ell}\left|z_{i}\right|+\max _{\ell+1 \leq j \leq 2 \ell}\left|z_{j}\right|\right\} \cdot\|\|_{A}^{T} \|_{1} .
$$

Observing that $\|A\|_{1}=\|T\|_{1}=2$ by construction, the result follows immediately from (5).

Proposition 2 below provides an easily computed sufficient condition for robust stability of the matrix polytope $L(\tilde{Z})$.

\section{Proposition 2}

If $\delta_{0}^{c} / 4$

$\leq\left\|A^{\mathrm{T}}\right\|_{1} \cdot \max _{1 \leq i \leq n} M_{i}^{-1} \cdot\left\{\max _{1 \leq j \leq \ell}\left(z_{j}-z_{j}^{c}\right)+\max _{1 \leq k \leq 2 \ell}\left(z_{k}-z_{k}^{c}\right)\right\} \cdot\|\|_{1}\left\|H^{-1}\right\|_{1}$

then the matrix polytope $L(Z)$ is robustly stable.

\section{Proof}

This follows directly from Lemma 1 and an eigenvalue perturbation result due to Bauer and Fike, reported in [4], pp. 87-88.

Note that given a fixed center point $\mathbf{L}\left(\mathbf{z}^{c}\right)$, if $\mathbf{L}\left(\mathbf{z}^{c}\right)$ is stable, and the hyper-rectangle $Z$ is sufficiently small, the hypothesis of Proposition 2 must hold. This will prove important in considering the convergence of the algorithm proposed in Section V.

\section{Sufficient Condition for Matrix Polytope Instability}

If $\mathbf{L}\left(\mathbf{z}^{\mathfrak{c}}\right)$ is stable, there exists a point $\hat{Z} \in \mathbb{Z}$ such that $\mathbf{L}(\hat{2})$ is unstable, it follows by continuity arguments that there exists a $\hat{\mathbf{z}} \in \tilde{\mathbf{Z}}$ such that $\mathbf{L}(\hat{\mathbf{z}})$ has an imaginary eigenvalue $j \omega$, with corresponding eigenvector $\mathbf{v}=\mathbf{a}+\mathbf{j b}$. To locate this $\hat{z}$, consider an augmented $2 n \times 2 n$ matrix function $L(z, \omega)$, defined as

$$
L(z, \omega)=\sum_{i=1}^{2 \ell+1} z_{i}\left[\begin{array}{cc}
L^{i} & 0 \\
0 & \mathbf{L}^{i}
\end{array}\right]+\omega\left[\begin{array}{cc}
0 & -I \\
I & 0
\end{array}\right]
$$

It will prove convenient to again expand the vector $\mathrm{z}$ and hyperrectangle $z$ to include $\omega$ as its $2 \ell+2$ component, with $L^{2 \ell+2}$ defined as the constant skew symmetric matrix multiplying $\omega$ above. The new $2 \ell+2$ dimensional hyper-rectangle will be denoted $z$. An interval for the $\omega$ axis of $z$ can be easily calculated by bounding the largest spectral radius expected for matrices in the original $L(\widetilde{Z})$. Letting

$$
\mathbf{x} \triangleq\left[\mathbf{a}^{\mathrm{T}}, \mathbf{b}^{\mathrm{T}}\right]^{\mathrm{T}},
$$

it follows that the polytope contains a matrix having $\mathrm{j} \omega$ as an eigenvalue if and only if there exists a $z \in Z$ and a $x \in R^{2 n}$, $\mathbf{x} \neq \mathbf{0}$, such that $\mathbf{L}(\mathbf{z}) \mathbf{x}=\mathbf{0}$. Establishing instability through this rank test is similar to the guardian map concept of [5], though the dimension of the augmented matrices is considerably smaller in the approach given here. From the rank test a condition for instability may be obtained as the solution to an optimization problem. Given $\mathbf{L}\left(\mathbf{z}^{\mathfrak{c}}\right)$ is stable, the matrix polytope $\mathbf{L}(Z)$ is unstable if and only if

$$
\min _{z \in Z,\|\mathbf{x}\|=1} \vartheta(\mathbf{x}, \mathbf{z}) \triangleq \mathbf{x}^{T} \mathbf{L}^{T}(z) \mathbf{L}(\mathbf{z}) \mathbf{x}=0
$$

In the construction above, note that there will exist constant matrices $\mathbf{N}^{i}, i=1,2, \ldots 2 n$ with the property that

$$
\sum_{i=1}^{2 \ell+2} z_{i} L^{i} x=\sum_{i=1}^{2 n} x_{i} N^{i} z \triangleq N(x) z
$$

The objective function therefore has the attractive property of being quadratic in the $\mathbf{x}$ and $\mathbf{z}$ variables individually. While this does not make the overall objective function convex in $(\mathbf{x}, \mathbf{z})$, it does suggest that local minima may be located by alternately minimizing with respect to one variable while the other is held fixed.

If this "alternating minimization" approach is to yield a local minima for the full cost function, it must be true that if the necessary Kuhn-Tucker conditions for minima are satisfied with respect to $\mathbf{x}$ and $\mathbf{z}$ individually, then the corresponding condition (gradient of cost orthogonal to tangent space of constraint manifold) for the overall problem is satisfied. To establish this result, the following notation is developed. Let $(\hat{\mathbf{x}}, \hat{\mathbf{z}})$ be a "candidate" local minimizer. The function $\mathrm{g}_{1}: \mathbf{R}^{2 \mathbf{n}} \rightarrow \mathbf{R}$ will denote the equality constraints on $\mathbf{x}$; i.e., $g_{1}(x) \triangleq\|x\|-1=0$. Let $g_{2}: \mathbf{R}^{2 \ell+2} \rightarrow \mathbf{R}^{\mathrm{m}}$ denote the inequality constraints active at $\hat{\mathbf{z}}$. For simplicity, we will assume that $\hat{\mathbf{z}}$ is not a vertex of the hyperrectangle $\mathcal{Z}$, so the constraints have a well defined tangent space at the point $(\hat{\mathbf{x}}, \hat{\mathbf{z}})$. We partition the composite vector $(\hat{\mathbf{x}}, \hat{\mathbf{z}})$ as independent variables $\hat{\mathbf{u}}$, and dependent variables $\hat{\mathbf{w}}$. The component $\hat{\mathbf{w}}$ will have dimension equal to the number of active constraints in $\mathrm{g}_{2}$, plus one for the $\mathrm{g}_{1}$ constraint.

The proposition to follow will use the reduced gradient [6]. Note that the selection of the dependent variable vector $w$ must be such that

$$
\operatorname{det} \frac{\partial g}{\partial \mathbf{w}}(\hat{\mathbf{x}}, \hat{\mathbf{w}}) \neq 0
$$

For the g's defined here, this implies that one component of $\mathbf{w}$ must be chosen from among the elements of $x$, say $x_{k}$, while the remaining components of $w$ are selected from elements of $z$. Assume that these components are ordered in $w$ with the $\mathbf{x}_{\mathbf{k}}$ element first and $\mathrm{z}$ elements following. Similarly, assume that the 
first $2 n-1$ components of $\mathbf{u}$ correspond to elements of $\mathbf{x}$, followed by components corresponding to $\mathrm{z}$. Let

$$
\begin{aligned}
R G[\vartheta]_{(\mathbf{x}, \mathbf{z})} \triangleq & \text { "Full" reduced gradient }= \\
& \frac{\partial \vartheta}{\partial \mathbf{u}}-\frac{\partial \vartheta}{\partial \mathbf{w}}\left[\frac{\partial \mathbf{g}}{\partial \mathbf{w}}\right]^{-1} \frac{\partial \mathbf{g}}{\partial \mathbf{u}}
\end{aligned}
$$

Next, consider the reduced gradient associated with the optimization problem in $\mathbf{x}$ alone, when $\mathbf{z}$ is held fixed. For this problem, only the $g_{1}(\mathbf{x})$ constraint is considered. Let $\mathbf{u}_{(\mathbf{x})}$ and $w_{(x)}$ denote the vectors obtained by retaining only those components of the independent and dependent variables corresponding to $\mathbf{x}$ terms (note that $\mathrm{w}_{(\mathrm{x})}$ will be a scalar). The notation $R G[\vartheta]_{(x)}$ will be used to represent the reduced gradient in this case, with

$$
R G[\vartheta]_{(\mathbf{x})}=\frac{\partial \vartheta}{\partial \mathbf{u}_{(\mathbf{x})}}-\frac{\partial \vartheta}{\partial \mathrm{w}_{(\mathbf{x})}}\left[\frac{\partial \mathrm{g}_{1}}{\partial \mathrm{w}_{(\mathbf{x})}}\right]^{-1} \frac{\partial \mathrm{g}_{1}}{\partial \mathrm{u}_{(\mathbf{x})}}
$$

Similarly, for the optimization problem in $\mathbf{z}$ alone, with $\mathbf{x}$ held fixed, let $\mathbf{u}_{(z)}$ and $\mathrm{w}_{(\mathbf{z})}$ denote the vectors obtained by retaining only those components corresponding to $\mathbf{z}$ terms. Then

$$
R G[\vartheta]_{(\mathrm{z})}=\frac{\partial \vartheta}{\partial \mathbf{u}_{(\mathrm{z})}}-\frac{\partial \vartheta}{\partial \mathbf{w}_{(\mathrm{z})}}\left[\frac{\partial \mathrm{g}_{2}}{\partial \mathbf{w}_{(\mathrm{z})}}\right]^{-1} \frac{\partial \mathbf{g}_{2}}{\partial \mathbf{u}_{(\mathrm{z})}}
$$

The desired result can be summarized in the following proposition.

\section{Proposition 3}

$$
\text { If } R G[\vartheta]_{(\mathbf{x})}(\hat{\mathbf{x}}, \hat{\mathbf{z}})=\mathbf{0} \text { and } R G[\vartheta]_{(\mathbf{z})}(\hat{\mathbf{x}}, \hat{\mathbf{z}})=\mathbf{0} \text {, then }
$$
$R G[\vartheta]_{(\mathbf{x}, \mathbf{z})}(\hat{\mathbf{x}}, \hat{\mathbf{z}})=\mathbf{0}$.

\section{Proof}

This result follows directly from the definition of the reduced gradient in each case, and the fact that for the "full" set of constraints, the partial of $\mathbf{g}$ with respect to $\mathbf{w}$ then has a block diagonal structure,

$$
\frac{\partial g}{\partial \mathbf{w}}(\hat{\mathbf{x}}, \hat{\mathbf{z}})=\left[\begin{array}{cc}
\frac{\partial \mathrm{g}_{1}}{\partial \mathrm{x}_{k}}(\hat{\mathbf{x}}) & 0 \\
0 & \frac{\partial \mathbf{g}_{2}}{\partial \mathbf{z}}(\hat{z})
\end{array}\right] .
$$

To exploit this result and provide a sufficient condition for instability of the polytope, the following "conceptual" algorithm is suggested:

0 ) Given feasible points $x^{0},\left\|x^{0}\right\|=1$, and $z^{\mathfrak{c}} \in Z$, with $L\left(z^{c}\right)$ assumed stable (again, if not, the polytope is trivially classified as unstable). Initialize index $i=0$.

$$
\text { While }\left\{R \mathcal{R}[\vartheta]_{(\mathbf{x})}\left(\mathbf{x}^{\mathrm{i}}, \mathbf{z}^{\mathrm{i}}\right) \neq \mathbf{0} \text { or } \mathscr{R} \mathcal{G}[\vartheta]_{(\mathbf{z})}\left(\mathbf{x}^{\mathrm{i}}, \mathbf{z}^{\mathrm{i}}\right) \neq \mathbf{0}\right\} \text {, do }
$$

1) With $z^{i}$ fixed, solve for $x^{i+1}$ to minimize: $\min _{\|\mathbf{x}\|=1} \mathbf{x}^{\mathrm{T}} \mathbf{L}^{\mathrm{T}}\left(\mathbf{z}^{\mathrm{i}}\right) \mathbf{L}\left(\mathbf{z}^{\mathrm{i}}\right) \mathbf{x}$

(note that for the Euclidean norm on $\mathbf{x}$, the solution is obtained directly from the singular value decomposition of $\mathbf{L}\left(\mathbf{z}^{\mathrm{i}}\right)$ );

2) With $x^{i+1}$ fixed, solve for $z^{i+1}$ to minimize: $\min _{z \in z} z^{\mathrm{T}} \mathbf{N}^{\mathrm{T}}\left(\mathbf{x}^{\mathrm{i}+1}\right) \mathbf{N}\left(\mathbf{x}^{\mathrm{i}+1}\right) \mathbf{z}$

3) $i=i+1$

\} .

If this algorithm terminates, Proposition 3 ensures that the resulting $(\hat{\mathbf{x}}, \hat{\mathbf{z}})$ point will satisfy the necessary condition for a local minimum of the overall optimization problem. If the cost function reaches zero at this point, or if $L\left(\mathbf{z}^{\mathfrak{C}}\right)$ is unstable at the outset, the polytope is unstable. If the algorithm fails to converge, or reaches a point with cost greater than zero, no conclusion can be drawn. However, the attractive feature of this algorithm is the simple nature of the optimization to be performed at steps 1 and 2. Step 1 is directly solvable from a singular value decomposition, while step 2 can be formulated as a standard quadratic program.

As in the test of Section III, an important issue relating to this algorithm is its performance as the polytope in question gets sufficiently small. Suppose $(\hat{\mathbf{x}}, \hat{\mathbf{z}})$ is a feasible point at which $\vartheta$ achieves its global minimum of $\vartheta(\hat{\mathbf{x}}, \hat{\mathbf{z}})=0$. We will assume that there exists a neighborhood of $(\hat{\mathbf{x}}, \hat{\mathbf{z}})$ with the property that any initial $\left(\mathbf{x}^{0}, \mathbf{z}^{0}\right)$ selected from that neighborhood will converge to $(\hat{\mathbf{x}}, \hat{\mathbf{z}})$ under the algorithm above. Without specifying in detail the optimization schemes to be used at steps 1 and 2 above, this local convergence condition cannot be formally proven here. However, it represents a reasonable minimum requirement for an iterative optimization scheme applied to a $C^{\infty}$ objective function. It will guarantee that for a sufficiently small hyper-rectangle $z$, if the polytope $\mathrm{L}(Z)$ contains a matrix having imaginary axis eigenvalues, then the algorithm above will converge to a point having zero cost and classify the polytope as unstable.

\section{Branch and Bound Robust Stability Test}

Given sufficiency tests for robust stability and instability of the matrix polytope, an overall necessary and sufficient test can be obtained by combining these in a "branch and bound" framework. Given data will consist of a hyper-rectangle $Z$, and corresponding $\mathrm{L}^{\mathrm{i}}$ 's describing a matrix polytope via (4). Consider a logical function $\mathcal{T S}(Z)$ that returns "true" if the polytope is robustly stable, and "false" if it is unstable. Using the tests outlined in sections III and IV, the following algorithm defines this function recursively: 
If the polytope $L(Z)$ satisfies the sufficient condition for robust stability of Section III, then $\mathcal{T S}(Z)=$ "true;"

else

if the polytope $\mathrm{L}(Z)$ satisfies the sufficient condition for instability of Section IV, then $T \mathcal{S}(Z)=$ "false;"

else

create new hyper-rectangles $z^{\mathrm{A}}$ and $z^{\mathrm{B}}$ by halving the longest axis of $Z$, and let

$\mathcal{T S}(Z)=\mathcal{T S}\left(Z^{\mathrm{A}}\right)$ and $\mathcal{T S}\left(Z^{\mathrm{B}}\right)$.

Note that at each "branching" step, the hyper-rectangle is halved along its longest axis. This ensures that for any $\varepsilon>0$, a sufficient number of branching steps will produce hyperrectangles with all axes less than $\varepsilon$ in length. Given the observations at the ends of Sections III and IV, once the hyperrectangles are sufficiently small, one or the other of the tests is guaranteed to yield a definitive conclusion for each corresponding matrix polytope.

More interesting is the computational cost of such an approach. In the worst case, the cost of computation can obviously grow exponentially. However, the test for instability offers the possibility for considerable computational savings, since if any one sub-polytope yields an unstable point, the algorithm terminates. Initial computational experience with a similar formulation [7] has proven promising for a number of matrix polytope test cases. The optimization successfully identified marginally stable matrices (i.e., the algorithm terminated at a zero cost point) in all unstable polytopes tested, without the need to divide these into smaller sub-polytopes. However, the test cases examined were of small dimension, and hardly exhaustive. A thorough computational test of the proposed algorithm applied to power system examples of reasonable dimension awaits further research efforts.

\section{Conclusions}

This paper has proposed a formulation representing a family of linearizations in a power system dynamic model as a polytope of matrices. Using this polytope structure, a simple sufficient condition for robust stability of the matrix polytope is developed. Also, an optimization scheme is proposed to provide a sufficient condition for instability of the polytope. These two tests are combined in a branch and bound algorithm that divides the polytope into smaller and smaller sub-polytopes, repeatedly testing for instability or robust stability. The algorithm terminates when any sub-polytope proves unstable, or when all sub-polytopes prove robustly stable. While the algorithm awaits further tests to ascertain its average computational cost in practice, the initial experience appears promising.

\section{References}

[1] N. Uchida and T. Nagao, "A New Eigen-analysis Method of Steady State Stability Studies for Large Power Systems: S Matrix Method," IEEE Trans. on Power Systems, vol. 3, pp. 706-714, May 1988.

[2] L. O. Chua, C. A. Desoer, and E. S. Kuh, Linear and Nonlinear Circuits, McGraw-Hill, New York, 1987.

[3] B. R. Barmish and C. L. DeMarco, "Criteria for robust stability of systems with structured uncertainty: A perspective," Proc. 1987 American Control Conference, pp. 476-481, Minneapolis, MN, June 1987.

[4] Wilkinson, J. H., The Algebraic Eigenvalue Problem, Oxford University Press, London, 1965.

[5] L. Saydy, A. L. Tits and E. H. Abed, "Robust Stability of Linear Systems Relative to Guarded Domains," Proc. 27th Conf. on Decision and Control, pp. 544-551, Austin, Texas, 1988.

[6] D. Luenberger, Introduction to Linear and Nonlinear Programming, Addison-Wesley, 1973.

[7] C. L. DeMarco, B. R. Barmish, and S. J. Saleh, "A Computational Approach to Establish Instability of a Polytope of Matrices," Memorandum no. ECE-88-11, Department of Electrical and Computer Engineering, University of Wisconsin-Madison, May 1988. 